\title{
Linx
}

Revue des linguistes de l'université Paris X Nanterre

$81 \mid 2020$

Enseignement des langues : la grammaire et la linguistique sont-elles solubles dans la pédagogie de projet?

\section{Appropriation de formes syntaxiques complexes à l'oral : constats et propositions}

Internalizing Complex Syntactic Structures in Oral Communication: findings and proposals

Pascale Goutéraux

\section{OpenEdition}

1 Journals

Édition électronique

URL : http://journals.openedition.org/linx/6868

DOI : $10.4000 /$ linx.6868

ISSN : 2118-9692

Éditeur

Presses universitaires de Paris Nanterre

Édition imprimée

Date de publication : 15 décembre 2020

ISSN : 0246-8743

Référence électronique

Pascale Goutéraux, « Appropriation de formes syntaxiques complexes à l'oral : constats et

propositions », Linx [En ligne], 81 | 2020, mis en ligne le 15 décembre 2020, consulté le 29 janvier 2021. URL : http://journals.openedition.org/linx/6868 ; DOI : https://doi.org/10.4000/linx.6868

Ce document a été généré automatiquement le 29 janvier 2021.

Département de Sciences du langage, Université Paris Ouest 


\title{
Appropriation de formes syntaxiques complexes à l'oral : constats et propositions
}

Internalizing Complex Syntactic Structures in Oral Communication: findings and proposals

\author{
Pascale Goutéraux
}

\section{Introduction}

Cet article interroge la reconnaissance et l'appropriation de structures complexes dans la communication orale en anglais langue étrangère. Pauvreté lexicale ou nonreconnaissance phonologique sont souvent évoquées comme les obstacles premiers à la compétence de réception orale des non-natifs. L'hypothèse ici explorée est que certains usages syntaxiques et grammaticaux de l'anglais oral sont à l'origine de hiatus communicationnels entre natifs et non-natifs et peuvent limiter la compétence de réception des apprenants d'anglais langue étrangère. L'étude présentée a porté sur les phénomènes d'intercompréhension, ajustement et réajustement discursif, développés par Pennec $(2012,2017)$ dans son analyse du discours anglophone, appliqués au corpus DIDEROT-LONGDALE (en cours de publication sur ORTOLANG), un corpus longitudinal de conversations entre locuteurs natifs, assistants d'anglais, et apprenants avancés, étudiants anglicistes de L1, L2 et L3 de l'université Paris-Diderot (Université de Paris), compilé de 2009 à 2013. Cette recherche a exploré l'hypothèse que les difficultés de compréhension manifestées par des étudiants anglicistes de Licence de niveaux B2/C1 du Cadre Européen Commun de Référence pour les Langues (désormais CECRL) (Conseil de l'Europe, 2001) n'étaient pas seulement dues à la méconnaissance d'éléments lexicaux ou d'expressions idiomatiques, à des difficultés de perception aurale, au manque d'attention des non-natifs aux formes non-saillantes ou non familières et à la plus faible capacité de leur mémoire de travail en langue étrangère (Gaonac'h \& Larigauderie, 2000), mais résultaient souvent de la non-reconnaissance de formes 
orales complexes produites par les assistants anglophones: interrogations noncanoniques ou peu fréquentes, anaphores et formes elliptiques, ou encore énoncés caractérisés par des propositions multiples. Après avoir analysé ces dysfonctionnements discursifs, l'article discute les stratégies d'inter-ajustement utilisées par les locuteurs natifs et les non-natifs pour résoudre ces hiatus conversationnels et renouer le fil de la conversation, tout en s'interrogeant sur leur efficacité à long terme pour la consolidation de la compétence linguistique. Comme l'indique l'analyse d'un échantillon de huit manuels de lycée récents, les apprenants ne sont guère explicitement exposés à ces formes syntaxiques peu saillantes au cours de leur cursus secondaire. La dernière partie propose des stratégies de conscientisation à mettre en œuvre pour la sensibilisation à certaines constructions syntaxiques spécifiques de l'oral.

2 La question de la spécificité et de la complexité des structures de l'oral fait débat. Tandis que certains analystes du discours, par exemple McCarthy (2001), soulignent la spécificité de la grammaire de l'oral, d'autres chercheurs comme Biber et al (1999), auteurs de la Longman Grammar of Spoken and Written English (LGSWE) ou Leech (2000) adoptent une position plus ambigüe: LGSWE par exemple pointe la simplification grammaticale en registre conversationnel tout en consacrant un chapitre (chap. 14) à la spécificité de la grammaire conversationnelle. La Nouvelle grammaire de l'oral (Huart, 2010) met en évidence les liens entre accentuation, saillance et phénomènes syntaxiques de l'oral (chap. 10 sur l'auxiliaire de reprise, chap. 13 sur les pronoms et leur emploi anaphorique, chap. 26 sur les phrases elliptiques). L'hypothèse ici explorée est que certains phénomènes linguistiques typiques de l'oral sont porteurs de complexité cognitive et linguistique pour l'apprenant de langue étrangère.

\section{Quelques définitions de la complexité}

3 La complexité 'cognitive' aussi dite 'relative' concerne le traitement de l'information, variable selon l'apprenant en termes de coût cognitif ou de difficulté, et en fonction du nombre d'opérations à effectuer sur l'énoncé, par exemple, les relatives, les passives (Bulté \& Housen, 2012, Housen et al, 2016). Il en est de même pour les propositions enchâssées ou les dislocations. Bulté et Housen ajoutent que relèvent de la complexité cognitive des occurrences qui ne sont pas 'objectivement' linguistiquement complexes mais sont peu saillantes à l'oral ou peu fréquentes. La complexité dite 'absolue' ou 'inhérente' est, elle, liée au nombre d'éléments dans l'énoncé et au nombre de connexions entre ces éléments. Elle comprend entre autres la complexité 'structurale ou linguistique' selon une définition minimale de la phrase complexe contenant «au moins deux propositions dont l'une joue un rôle syntaxique par rapport à l'autre " (Oriez, 2009 : 131). En acquisition de la langue étrangère, les notions de complexité et de difficulté se chevauchent parfois : par exemple la régulation intra-subjective que le locuteur natif opère sur son propre discours peut paradoxalement se révéler facteur de confusion pour l'interlocuteur non-natif qui peine à suivre les méandres du discours initial. Le concept de régulation intra-subjective a été développé par Pennec dans ses travaux sur l'ajustement et le réajustement discursif $(2012,2017)$. A titre d'exemple d'ajustement intra-subjectif, voici un énoncé complexe produit par un assistant britannique et la réponse de l'étudiant de L1 :

(1) 
$\mathrm{N}$ : so it's kind of like a a coming of age film or an adventure film is it? rather than a (er) as a kind of film it's like a coming of age sort of film about four teen sort of teenagers kind of fighting against er life-like teenagers' obstacles in order to get what they want?

[donc c'est un peu un film sur le le passage à l'âge adulte ou un film d'aventure, non? Plutôt qu'un (heu) c'est un film genre un peu sur le passage à l'âge adulte sur quatre types d'adolescents qui genre affrontent des obstacles typiques de l'adolescence pour obtenir ce qu'ils veulent?]

NN (L1): yes, it's a comic movie. It's er it's really something you watch er in family or with your friends.

[Oui, c'est un film drôle. C'est heu c'est vraiment un truc que tu regardes en famille ou avec des amis]

Ce long énoncé natif est ponctué de disfluences et d'inserts discursifs visant à gagner du temps de planification du discours ( 3 kind of, 2 like, 1 sort of) ; s'y ajoutent un tag interrogatif de demande de confirmation (is it?), une participiale, une proposition de but, une complétive (what they want) + un génitif complexe (er life-like teenagers' obstacles). L'énoncé illustre la façon dont le locuteur natif ajuste son discours à sa pensée et le réélabore pour préciser des formulations imparfaites. Le non-natif s'est accroché à deux bribes lexicales (com....movie) de l'expression coming of age movie (2 occurrences) mais a échoué à inférer le sens comme l'illustre le passage de 'coming of age movie' à 'comic movie'.

Une autre partie de cet article (paragraphe 3.5) traite plus spécifiquement de l'ajustement intersubjectif, qui porte sur la relation d'intercompréhension entre les locuteurs et les stratégies discursives utilisées par le natif pour résoudre si possible ce que Culioli appelle des phénomènes de «non-coïncidences du dire» entre interlocuteurs et assurer la continuité conversationnelle (2002:34, rapporté en 2009).

\section{Analyse du corpus DIDEROT-LONGDALE}

6 L'analyse a porté sur cent conversations semi-guidées, de longueur variable, de 7 à 13 minutes (soit un quart du corpus DIDEROT-LONGDALE de conversations entre étudiants anglicistes de Licence (niveau B2 à C1) et cinq assistants anglophones, 2 hommes et 3 femmes). Soixante conversations ne présentent aucun épisode flagrant d'incompréhension. Plusieurs explications peuvent être invoquées : le questionnement $\mathrm{du}$ locuteur natif est souvent minimal, canonique, ou proche des structures auxquelles les étudiants sont habitués dans l'enseignement secondaire. Certains étudiants parviennent à reconstituer le sens en prélevant suffisamment d'éléments sémantiques significatifs dans le discours du natif. Une expérience d'immersion en pays anglophone facilite parfois la compréhension d'expressions idiomatiques utilisées par les natifs.

Quarante conversations présentent un ou plusieurs épisodes d'incompréhension, de la part des non-natifs, soit un total de 53 épisodes : 51 en L1 dont 36 en première session de septembre (S001), 15 en deuxième session de juin (S002), 2 seulement en L3 (S004). Ils sont signalés par 49 marqueurs lexicaux, 11 marques extralinguistiques (interjections, pauses pleines, rires embarrassés) et parfois des réponses incongrues sans cohérence avec la question initiale. Le logiciel Wordsmith7 (Scott, 2016) a permis de repérer les marqueurs témoignant de phénomènes d'incompréhension et d'ajustement. Le hiatus communicationnel s'exprime par des pauses, vides ou pleines ( $\mathrm{hu}, \mathrm{er}, \mathrm{em}, \mathrm{erm}$ ) associées à des amorces interrogatives de type what? (11) ou de quoi?, des aveux d'incompréhension (I don't understand, I don't know this sentence), des demandes de 
répétition (Can you repeat, please ?,repeat, please, can you repeat the question (2) ou encore sorry (8), excuse me (1).

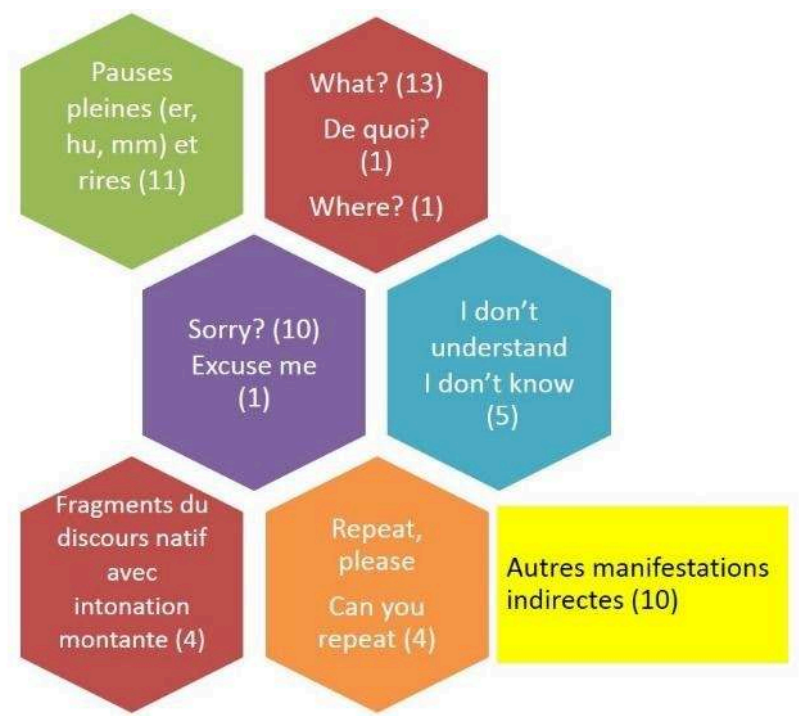

Figure 1 : Marqueurs d'incompréhension dans le discours non-natif

La fonction Concord de Wordsmith7 a permis d'intégrer le contexte antérieur et postérieur de certains phénomènes linguistiques complexes, de récupérer les tours de parole de l'interlocuteur natif déclencheurs de confusion ainsi que les stratégies d'ajustement et de réparation proposées. Cette méthode à la fois quantitative et qualitative facilite la localisation rapide des énoncés déclencheurs de rupture conversationnelle. Un balayage manuel a été indispensable pour identifier les pauses pleines porteuses d'incompréhension (les pauses vides n'ont été comptabilisées que si elles étaient significatives) ainsi que les réponses incongrues; d'autres confusions ont été repérées, par exemple entre why et when.

(2)

$\mathrm{N}$ : why did you decide to go to drama school?

$\mathrm{NN}$ : * two years ago er three years ago.

[N: pourquoi t'as voulu faire une école de comédie?

NN: il y a deux ans heu trois ans.]

\section{Enoncés complexes déclencheurs d'incompréhension}

Plusieurs types d'énoncés complexes produits par les locuteurs natifs se sont révélés problématiques pour les interlocuteurs non-natifs. Exprimés en pourcentage du total d'énoncés natifs qui posent problème aux non-natifs (ou épisodes d'incompréhension), ils mettent en lumière la fréquence des difficultés de compréhension dont ils sont porteurs :

Des questions peu usuelles (préposition en fin d'énoncé, tags de confirmation, formules indirectes, modalité interrogative portée par l'intonation) (36\%)

- Des problèmes de référence : anaphores et ellipses (24\%)

- Des énoncés longs avec multiplicité de propositions (19\%)

- Des expressions idiomatiques opaques (13\%)

- Autres : par exemple segmentation phonologique erronée du récepteur (8\%). 
11 Les paragraphes suivants développent les spécificités des formes linguistiques génératrices d'incompréhension.

\subsection{Formes interrogatives complexes}

La non-reconnaissance de certaines formes interrogatives a généré des épisodes d'incompréhension; il s'agit par exemple d'énoncés déclaratifs associés à des reprises, de questions indirectes ou directes ou de questions avec prépositions en fin de phrase (ex: what....like), des cas où l'apprenant ne peut opérer de transfert positif depuis le français puisque la structure n'existe pas dans cette langue; on trouve aussi des énoncés négatifs à intonation montante dont la valeur interrogative implicite est difficile à appréhender pour le non-natif. Voici quelques exemples de formes interrogatives complexes problématiques pour les non-natifs.

\subsubsection{Interrogatives avec préposition en fin d'énoncé ( 7 occurrences)}

(3) (S001)

$\mathrm{N}$ : Who did you go with (...) who did you go with?

$\mathrm{NN}$ : er er (échec)

[N: Tu y es allé avec qui (...) tu y es allé avec qui?

NN: heu heu ]

(4) (S002- fin de L1)

$\mathrm{N}$ : which cities were you disappointed about?

$\mathrm{NN}: m m$

$\mathrm{N}$ : which which cities did you find disappointing? (reformulation/simplification)

NN: Melbourne (ajustement)

[N: Quelles sont les villes qui t'ont déçu?

$\mathrm{NN}$ : $\mathrm{mm}$

$\mathrm{N}$ : Quelles villes as-tu trouvé décevantes?

NN: Melbourne]

\subsubsection{Autres formes complexes de questionnement (7 occurrences)}

(5) (S002) question directe en how

$\mathrm{N}$ : and I also wanted to ask you (er) about the first year last year at Charles Cinq how was it

for you?

$\mathrm{NN}:$... What?

[N: et je voulais aussi te demander (heu) des détails sur ta première année à Charles 5 ça s'est apssé comment pour toi?

NN: ... Quoi?]

(6) Structure indirecte en how

$\mathrm{N}$ : $\mathrm{mm}$ so tell me how it happened that you em came to work with Martin Scorsese

$\mathrm{NN}$ : sorry?

$\mathrm{N}$ : how how did you end up working with Martin Scorsese? How did that happen?

NN: oh I I actually I haven't

[n: Bon alors dis moi comment ça se fait que tu as travaillé avec Martin Scorcese

NN: Pardon?

$\mathrm{N}$ : Comment t'es-tu retrouvé à travailler avec Martin Scorsese? Comment c'est arrivé?

NN: Ah, je je en fait je ne l'ai pas fait]

La déconstruction de l'énoncé et sa reformulation par l'assistant par deux questions directes aide le non-natif à surmonter la difficulté (complexité) cognitive et appréhender le sens global de la structure linguistique. 
14 L'exemple suivant concerne des reprises avec tags de confirmation et illustre le fait que la suppression du tag - stratégie adoptée par le natif - permet à l'apprenant de surmonter l'incompréhension initiale.

(7) tags

$\mathrm{N}$ : right so you're more more disappointed in yourself than actually in in the program was

that it?

$\mathrm{NN}$ : er excuse me

$\mathrm{N}$ : you were more disappointed in yourself than in the program?

NN: yes

[N: d'accord donc tu es plus déçu par toi même que par le programme en fait, c'est

ça?

NN: heu pardon

$\mathrm{N}$ : tu as été plus déçu par toi-même que par le programme?

NN: oui]

\subsubsection{Conflit linguistico-cognitif : énoncé négatif et intonation montante (5 occurrences)}

Dans l'échange ci-dessous, le mot rides (les attractions) semble connu, de même que les expressions ride the rides ou go on the rides puisque l'étudiant travaille à Disneyland Paris. C'est la forme du questionnement avec structure négative et intonation montante à valeur interrogative qui pose problème. On peut penser que la visée humoristique du locuteur qui sous-entend que l'étudiant n'est plus un enfant qui monte sur les manèges échappe à ce dernier. La simplification du questionnement par une question directe canonique permet la résolution du conflit linguistico-cognitif par le non-natif mais aux dépends de sa compréhension de l'humour du natif.

(8)

NN: but now I work in *Disneylands on the weekend so (rit) yes I guess I go to Disneyland

very often but er yeah

$\mathrm{N}$ : but you don't ride the rides anymore? (rit)

NN sorry?

$\mathrm{N}$ : do you still go on the on the rides?

$\mathrm{NN}$ : yes when I have *break sometimes

[NN: mais maintenant je travaille à Disney le weekend donc (rit) oui j'imagine que je

vais à Disney très souvent mais heu oui

$\mathrm{N}$ : mais tu ne fais plus de manège? (rit)

NN: pardon?

$\mathrm{N}$ : tu vas toujours sur les manèges?

NN: oui quand j'ai une pause parfois]

L'exemple 9 confirme la difficulté pour les non-natifs de réconcilier structure négative et visée interrogative portée par l'intonation montante.

(9)

$\mathrm{N}$ : but that doesn't stop you from wanting to live there $\uparrow$

$\mathrm{NN}$ : sorry?

$\mathrm{N}$ : but you'd still like to live there $\uparrow$

$\mathrm{NN}$ : er yeah because I met some people *very open minded

[N: mais ça ne t'empêche pas d'avoir envie d'y vivre $\uparrow$

NN: pardon?

$\mathrm{N}$ : mais tu voudrais quand même y vivre $\uparrow$

$\mathrm{NN}$ : heu ouais parce que j'ai rencontré des gens très ouverts] 


\subsection{Problèmes de référence : anaphore et ellipse}

17 Les problèmes d'identification de la référence (9 épisodes) sont un deuxième facteur de complexité cognitive et une cause d'incompréhension chez les sujets du corpus DIDEROT-LONGDALE, notamment lorsque le référent n'est pas immédiatement récupérable en mémoire de travail. Dans l'exemple 10, le référent nominal est localisé dans un tour de parole antérieur de l'étudiant qui raconte son expérience au Canada. Après une digression narrative, l'assistant revient sur les circonstances du voyage au Canada et interroge l'étudiant sur ses activités là-bas. La pause pleine exprime l'embarras de l'étudiant et les deux derniers tours montrent qu'il est nécessaire que l'assistant réintègre le référent nominal de there pour que l'étudiant associe le pays et l'élément anaphorique.

(10)

$\mathrm{N}$ : erm and what did you do whilst you were there?

$\mathrm{NN}: m m$

$\mathrm{N}$ : what did you do whilst you were in Canada?

$\mathrm{NN}$ : oh I visited a lot of ${ }^{*}$ thing

[N: hum, et qu'est-ce que tu as fait là-bas?

$\mathrm{NN}: \mathrm{mm}$

$\mathrm{N}$ : qu'est-ce que tu as fait quand tu étais au Canada?

$\mathrm{NN}$ : oh j'ai visité plein de choses]

Un schéma semblable se retrouve dans l'exemple 11. Le premier tour de parole de l'étudiant contient bien le référent nominal Potsdam (la ville) repris par l'assistant avec l'anaphorique there. Par la suite, l'étudiant n'associe plus there au référent implicite Potsdam, ce qui requiert une expansion explicite de l'assistant (in the city) pour réactiver le référent en mémoire de travail de l'apprenant. La réponse finale témoigne-t-elle d'un ajustement réussi ? Il n'est pas évident que la découverte d'un squat soit une activité touristique préférée.

(11)

$\mathrm{NN}$ : I don't know I don't remember the I think it was in Potsdam too so just Potsdam

$\mathrm{N}$ : $m m$ what were some of your favorite things that you guys did while you were there?

$\mathrm{NN}: \mathrm{em}$

$\mathrm{N}$ : in the city

$\mathrm{NN}$ : em we em we discovered a a squat can we say that yes a squat.

[NN: je ne sais pas je ne me souviens pas le je pense que c'était à Potsdam

$\mathrm{N}$ : mm et quels sont les trucs que vous avez faits que vous avez préféré quand vous

étiez là-bas?

NN: em

$\mathrm{N}$ : dans cette ville

$\mathrm{NN}$ : heu on heu on a découvert un squat est-ce qu'on peut dire ça oui un squat]

Les formes elliptiques sont tout aussi problématiques pour la compréhension mais comme pour l'anaphore, la réintégration de l'information explicite manquante (disponible dans le contexte discursif avant) facilite la reprise du flux conversationnel.

(12) Un voyage aux USA (S004)

$\mathrm{N}$ : and er did your university make it easy for you to go away?

$\mathrm{NN}$ : to go away?

$\mathrm{N}$ : to go to America?

$\mathrm{NN}$ : ah yeah.

[N: et heu est-ce que ta fac t'a aidé à partir?

$\mathrm{NN}$ : à partir?

$\mathrm{N}$ : à partir en Amérique? 
$\mathrm{NN}$ : ah ouais]

Enfin certains épisodes illustrent les effets de multiples formes complexes cumulées : dans l'exemple 13, l'étudiant ne comprend pas que le référent situationnel de that est 'être logé dans une auberge de jeunesse'; il est aussi déconcerté par la reformulation en what...like par l'assistant qui l'interroge sur son ressenti de l'expérience. Comme le suggère sa réplique finale, l'étudiant témoigne d'une compréhension très littérale avec une amorce de description concrète de l'auberge de jeunesse (it's like a big erm...).

(13)

$\mathrm{N}$ : where did you stay when you were in Belfast?

$\mathrm{NN}$ : er we were in a youth er hostel yeah

$\mathrm{N}$ : okay was that all right?

$\mathrm{NN}$ : what?

$\mathrm{N}$ : what was that like?

$\mathrm{NN}$ : it's like a big erm I don't know

[N: t'as logé où à Belfast?

$\mathrm{NN}$ : heu on a pris une auberge de jeunesse

$\mathrm{N}$ : okay c'était correct?

$\mathrm{NN}$ : quoi?

$\mathrm{N}$ : c'était comment?

$\mathrm{NN}$ : c'est genre une grande heu je sais pas]

21 L'épisode 14 est aussi déclenché par une combinaison de phénomènes : ellipse de la principale, insertion de trois propositions complétives nominales et probablement méconnaissance de la structure what...like (en réponse à une demande de clarification de la consigne par l'étudiant).

(14)

$\mathrm{NN}$ : what do you want me me to explain actually the the

$\mathrm{N}$ : kinda what it's like and how it's different and how you feel about it

$\mathrm{NN}$ : er I *like the mentality is different

[NN: qu'est-ce que tu veux que j'explique en fait le le

$\mathrm{N}$ : genre comment c'est en quoi c'est différent et ton ressenti

$\mathrm{NN}$ : heu comme la mentalité est différente]

\section{3 Énoncés longs complexes}

$19 \%$ des épisodes d'incompréhension sont dus à la longueur de l'énoncé et à la multiplicité des propositions, qui causent une déperdition de l'attention de l'interlocuteur non-natif. Gaonac'h \& Larigauderie (2000) soulignent la difficulté pour l'apprenant de stocker en mémoire de travail des énoncés trop longs ou des items trop nombreux en langue étrangère ; Skehan (1998) évoque la surcharge cognitive (cognitive overload) de l'apprenant de langue étrangère. Dans certains cas, comme en 15, ce n'est pas la variété des structures syntaxiques mais leur multiplicité et la longueur de l'énoncé, 4 ou 6 structures selon que les groupes verbaux coordonnés (write down the story and then learn it by heart) sont comptés séparément ou non, qui posent problème et rendent l'énoncé cognitivement complexe (Housen et al, 2016).

(15)

$\mathrm{N}$ : so did you have to er learn the story? Did you have to write down the story and then learn it by heart and do it in front of the class or did you just have to go up there and start talking?

$\mathrm{NN}$ : er repeat please er 
[N: alors est-ce qu'il a fallu que heu tu apprennes l'histoire? Est-ce qu'il a fallu que tu écrives l'histoire et qu'ensuite tu l'apprennes par cœur et que tu la dises devant la classe ou tu es juste monté sur l'estrade et tu t'es lancé?

$\mathrm{NN}$ : heu répète s'il te plaît heu]

\section{4 Problèmes d'idiomaticité}

13\% seulement des épisodes d'incompréhension sont liés à la méconnaissance d'expressions idiomatiques utilisées par le locuteur natif. Contrairement à d'autres structures grammaticalement ou syntaxiquement complexes, ces formes lexicales sont plus opaques et leur résolution est souvent délicate et la reprise conversationnelle peu évidente. Ainsi dans l'exemple 16, l'étudiante qui n'est jamais allée en pays anglophone a le projet de visiter Londres avec son ami.

(16) (s002)

$\mathrm{N}$ : ok well don't let him do all the talking

$\mathrm{NN}$ : er can you repeat please?

$\mathrm{N}$ : don't let him do all the talking you have to speak as well when you're there

$\mathrm{NN}$ : er I don't understand

$\mathrm{N}$ : never mind.

[N: ok ben ne le laisse pas parler tout le temps

NN: heu tu peux répéter s'il te plaît?

$\mathrm{N}$ : ne le laisse pas parler tout le temps il faut que toi aussi tu parles quand tu seras

là-bas

$\mathrm{NN}$ : heu je comprends pas

$\mathrm{N}$ : c'est pas grave]

La méconnaissance de l'expression idiomatique do all the talking et l'incompréhension de l'intention humoristique bloquent la communication malgré la répétition et la reformulation de la locutrice américaine (you have to speak as well when you're there). Dans ce cas précis, il n'y a pas de réajustement intersubjectif et l'assistante clôt l'échange et bifurque vers une autre question.

\subsection{Stratégies de réajustement discursif intersubjectif}

Ce paragraphe analyse les stratégies utilisées par les locuteurs natifs pour résoudre les problèmes d'incompréhension des non-natifs liés aux problèmes syntaxiques (interrogation et référence) et cognitifs (longueur et multiplicité des énoncés) et pose la question de leur efficacité. Certains exemples (8 et 16) témoignent que le non-natif ne parvient pas à dépasser une interprétation littérale de la structure et à percevoir la visée pragmatique souvent humoristique de l'énoncé natif.

Les recherches plus anciennes en acquisition et communication exolingue (par exemple, De Pietro, Matthei et Pi, 1989, Gaonac'h, 1984) montrent que dans les interactions entre natifs et non-natifs en milieu naturel, l'enjeu premier est d'assurer négociation et compromis et que les natifs adaptent souvent pragmatiquement leur discours pour faciliter l'intercompréhension. On retiendra le concept de conduites adaptatives en cours de conversation entre apprenant et natif qui évoque le concept d'ajustement intersubjectif de Pennec pour le discours natif. La visée est communicationnelle au sens large, puisque le décentrage provisoire du message sur le code contribue à une meilleure fluidité et à une relance du flux discursif. Les stratégies intersubjectives adoptées par les natifs du corpus DIDEROT-LONGDALE sont, sur un 
continuum du moins fréquent au plus fréquent : les répétitions (rares), les expansions (ex. 11, 16), la simplification de la forme (ex. 4, 7), la reformulation et la simplification du contenu sémantique (ex. 17), qui s'avère la plus utile pour le maintien de la conversation. Dans un objectif communicationnel à court terme, le natif n'hésite pas à simplifier son propos par l'emploi d'une expression parfois sémantiquement éloignée de l'expression originelle.

(17)

$\mathrm{N}$ : What are your plans when you're done here with your degree?

$\mathrm{NN}$ : er

$\mathrm{N}$ : what would you like to do?

$\mathrm{NN}$ : I'd like to go to to live in a in a country well er America

[N: quels sont tes projets quand t'en auras fini avec ta licence?

$\mathrm{NN}$ : heu

$\mathrm{N}$ : qu'est-ce que tu aimerais faire?

NN: j'aimerais partir vivre dans un pays ben heu l'Amérique]

Les anglophones ont donc naturellement recours à des reformulations et des expansions, des stratégies implicites efficaces pour la négociation immédiate du sens plus que pour la consolidation de la compétence linguistique, bien que certains auteurs (par exemple Bitchener, 2004) considèrent que la focalisation incidente sur la forme participe de l'acquisition. La réintégration explicite de la référence et de l'information manquante par le natif suffit en général à résoudre ponctuellement les problèmes de compréhension liés à des phénomènes d'anaphore et d'ellipse. Néanmoins, on peut s'interroger sur l'efficacité de cette focalisation incidente pour l'appropriation de formes discursives complexes. Ces stratégies de contournement ou de simplification à visée communicationnelle et non linguistique bloquent l'appropriation de ces phénomènes linguistiques peu saillants dans la langue anglaise et limitent le développement de l'interlangue en tant que mode de construction de la compétence linguistique. Comme le souligne Bailly (1999: 54) qui s'inscrit dans une conception constructiviste de l'acquisition, il est indispensable de faire appel à la conscientisation des apprenants :

Le recours à la réflexion dans l'enseignement de L2 table sur le fait que l'apprenant

élabore pour lui-même des systèmes intermédiaires d'explication du

fonctionnement de la langue qu'il modifie au fur et à mesure de l'apprentissage.

\section{Méthodologie actionnelle et problèmes de complexité}

Comment l'enseignement-apprentissage de l'anglais à visée actionnelle aborde-t-il ces questions de complexité? Cette partie s'interroge sur leur traitement dans le CECRL, dans les manuels de lycée contemporains et dans les pratiques de classe.

\subsection{La complexité dans le CECRL}

Les textes officiels du Lycée depuis 2005 s'appuient sur les descripteurs du CECRL de 2001 (enrichis par le volume complémentaire de 2018). Les descripteurs des niveaux B2 à $\mathrm{C} 1$ applicables au cycle terminal insistent sur la capacité des apprenants au lycée à produire des structures complexes à finalité clairement pragmatique (CECRL, $2001: 27)$ : $\mathrm{Au}$ niveau B2, l'apprenant peut «communiquer avec un degré de spontanéité et 
d'aisance qui rende possible une interaction normale avec un interlocuteur natif ». Le volume complémentaire (2018) éclaire quelque peu cet énoncé (niveau B2). On note la capacité des apprenants à comprendre les idées principales d'un discours complexe du point de vue pragmatique et linguistique (je reformule). L'élève doit pouvoir suivre un discours étendu linguistiquement complexe si la direction du discours dans une langue standard est marquée par des marqueurs explicites; au niveau C1, l'apprenant sera capable de suivre un discours étendu qui n'est pas clairement structuré et dans lequel les relations sont implicites ou ne sont pas signalées explicitement. Longueur des énoncés, usage idiomatique et relations implicites non signalées explicitement entre les éléments semblent caractéristiques des difficultés de compréhension rencontrées par les étudiants de L1 du corpus DIDEROT-LONGDALE.

\subsection{Les manuels de lycée contemporains}

Les manuels scolaires contemporains constituent un corpus utile pour analyser les problématiques linguistiques abordées en enseignement-apprentissage en lien avec les programmes officiels. Bien que les enseignants de lycée tendent à construire leurs propres séquences, les manuels sont fréquemment utilisés comme ouvrages de référence dans lesquels l'enseignant, notamment le jeune professeur, sélectionne des activités, tâches et exercices pour élaborer des préparations didactiques personnalisées. Les élèves ont aussi un accès gratuit aux matériaux en ligne des méthodes retenues dans les différentes classes. Il est donc utile d'explorer comment les manuels de lycée du cycle terminal (niveau B2 visé) abordent ou revisitent ces phénomènes spécifiques du discours conversationnel authentique. L'analyse des pages méthodologiques et entraînements linguistiques de huit manuels récents (cf. bibliographie pour la liste complète, 2012-2016) donne quelques indications sur le traitement de ces structures conversationnelles complexes. Par exemple, seul skylight (2012) affiche une rubrique The Orality of Language et propose des pages intitulées Taskbased Grammar. Les questions rhétoriques, les tags, leur usage discursif et intonations sont explicitement mentionnées comme contribuant à l'accomplissement de tâches discursives orales. D'autres manuels présentent des tableaux récapitulatifs avec tags,

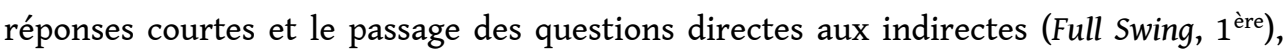
mais sans approche comparative/contrastive avec le français ni intégration aux tâches. New Mission, Terminale (2016) inclut des remarques sur l'intonation montante ou descendante des tags (réduite à l'intention pragmatique d'incertitude ou confirmation). Le précis de ce manuel 'Door to English' (partie 'les compléments du verbe') indique que les prépositions sont à droite du verbe et donne 3 exemples: une relative, une interrogative et une passive, mais ne propose aucune activité de manipulation, de réflexion ou de préparation à une tâche. On trouve aussi quelques exemples dans d'autres manuels d'interrogatives avec prépositions en fin d'énoncé. Concernant les questions d'anaphore et d'ellipse, seul le manuel New on Target, Terminale (2016) (section 'Language Corner : Omitting Words') définit une pro-forme comme un mot qui évite la répétition d'un autre mot ou d'une proposition entière, le lie à l'usage des tags et propose des repérages dans un extrait écrit et un enregistrement. Le précis de Diversity (2015, p. 218) comporte quelques lignes sur les pro-formes they et one et un exercice de complétude. Ces quelques remarques suggèrent que la grammaire conversationnelle ne semble pas être une priorité et que l'approche explicite des structures syntaxiques de l'oral est partielle dans les manuels de lycée. 


\subsection{Les pratiques de classe}

31 La persistance d'incompréhensions liées à la syntaxe de l'oral chez les apprenants avancés de Licence Etudes anglophones pourrait résulter paradoxalement de pratiques dans le secondaire qui privilégient la communication au sein de groupes d'élèves pour l'accomplissement de tâches actionnelles. Cette ébauche de réflexion mériterait d'être développée par l'analyse systématique de corpus d'interactions orales en classes d'anglais langue étrangère. En production orale interactive, le questionnement pratiqué dans le cadre de tâches porte sur des échanges d'information, des questionnaires élaborés par les élèves, des simulations d'entretiens, lesquels favorisent l'usage de questions simplifiées ouvertes et fermées, sans préposition en fin d'énoncé, ni tags, ni réponses courtes attendues. Les activités de réception menées en groupes ou au sein d'îlots selon le mode de la coopération entre élèves prôné par les instructions officielles privilégient la co-reconstruction du sens. L'accent porte sur ce que les apprenants ont compris d'un texte oral ou écrit et leur capacité à le reformuler dans leurs propres mots. Une approche cognitive systématiquement descendante, qui ne serait pas complétée par un processus ascendant de reconstruction sémantique à partir d'une analyse fine de la forme textuelle dirigée par le professeur, risque d'entraîner la minoration de ce qui n'est pas compris, ce qui est peu perceptible, ou le remplacement d'énoncés complexes par des reformulations simplifiées. Enfin, l'enseignant de langue étrangère est invité à limiter sa prise de parole, à remplacer les énoncés interrogatifs trop longs et complets par des amorces (prompts) brèves visant à favoriser l'expression de l'élève et son autonomisation dans le secondaire lorsque les formes interrogatives de base sont considérées comme acquises. L'absence de focalisation sur des énoncés présentant des structures spécifiques de la grammaire de l'oral en usage, qu'ils soient extraits de documents authentiques ou utilisés régulièrement par le professeur en communication orale, tend à oblitérer la conscientisation de ces formes complexes. Un enseignement inductif, de l'implicite à l'explicite, ou une démarche maïeutique de conscientisation (Bailly, 1999) sont nécessaires à l'appropriation des phénomènes linguistiques (temps, aspect, modalité et détermination) problématiques pour les francophones. Dans l'enseignement secondaire français, la réflexion sur la langue a fait partie des programmes depuis 1985 (un des trois objectifs) et des pratiques de classe (pratique raisonnée de la langue, puis réflexion sur la langue), notamment pendant la période communicative et cognitive jusque dans les années 2000. Depuis la valorisation de l'approche actionnelle et l'accent mis sur les tâches, la réflexion linguistique est passée à l'arrière-plan et les moments épisodiques de réflexion en classe portent le plus souvent sur des énoncés ponctuels (authentiques ou didactiques) et des traces écrites élaborées à partir de productions d'élèves, ce qui ne favorise pas le traitement d'interrogatives complexes ou de phénomènes d'anaphore et d'ellipse.

\subsection{Quelques pistes de réhabilitation des formes complexes en discours oral}

Les chercheurs anglophones en acquisition insistent sur la nécessité de mettre en évidence des formes linguistiques de faible saillance ou qui passent inaperçues lorsque l'attention de l'apprenant se porte sur d'autres phénomènes linguistiques plus 
familiers, plus transférables de sa L1 (N. Ellis, 2007: 30). Selon Schmidt (chapitre 'Attention', 2001: 23),

Since many features of L2 input are likely to be infrequent, non-salient and communicatively redundant, intentionally focused attention may be a practical (though not a theoretical) necessity for successful language learning.

[Dans la mesure où de nombreuses structures de la L2 sont susceptibles d'être rares, non saillantes et communicativement redondantes, diriger de façon intentionnelle l'attention des apprenants sur certains phénomènes peut se révéler pratiquement (mais non théoriquement) nécessaire à la réussite de l'apprentissage d'une langue étrangère.]

Comment réconcilier tâches et complexité linguistique? R. Ellis $(2003,2016)$ propose d'élaborer des tâches focalisées sur l'interlangue (focused tasks) en complément de tâches non ciblées (unfocused tasks) à visée sociale, pratique. Dans le secondaire, on pourrait travailler sur le décalage (Noticing the Gap Tasks) entre productions conversationnelles entre non-natifs et conversations entre natifs: partir de conversations entre élèves (par exemple dans le cadre d'une tâche), proposer des conversations entre locuteurs natifs sur la même thématique incluant des faits de langue complexes et amener les élèves à retravailler et enrichir leurs productions.

A titre d'application, cette dernière partie est consacrée à des pistes expérimentées ou ébauchées qui concernent la conscientisation de faits de langue non saillants cognitivement complexes comme les questions de coréférence. Certaines tâches peuvent être dédiées à la conscientisation de l'association forme-sens (Focus-on-Form activities) par soulignement, surlignage, mise en évidence typographie ( $c f$. White, 1998 : 107). Voici un exemple de réflexion sur la coréférence, soit la mise en relief des liens entre marqueurs anaphoriques et référents par des étudiants (plus élaborée que celle de White qui s'adressait à des débutants) à partir du conte The Frog Prince des frères Grimm. A partir du texte brut, les étudiants mirent en évidence les liens entre référents et pro-formes, gras et italiques pour la princesse et italiques pour le Prince-Grenouille, puis organisèrent les relations par un système de fléchage (non reproduit ici), qui favorisa la prise de conscience de la multiplicité des formes de coréférence et incidemment permit à certains de se remémorer le genre masculin de frog en anglais à partir des pronoms sujet et complément :

[...] "What's the matter?" Said a voice behind her. The girl looked around and she saw a frog. He was in the well, his head sticking out of the water.

"Oh it's you" said the girl. "My ball fell into the well".

"I can help" said the frog. "I can get your ball. What will you give me if I do?"

"Whatever you want" said the princess [...]

$\mathrm{He}$ continued "I would like to eat from your dish and drink from your cup. I would

like to sleep on your bed. If I get your ball, will you promise me all this, Princess?"

"Oh yes" said the princess. "I'll promise." But she thought, "Silly frog, I could never live with him."

When the frog heard her promise, he went down in the well. He soon came up with the golden ball in his mouth. He put it at her feet.

She was happy when she saw her ball. She picked it up and ran away.

[[...] 'Qu'est-ce qu'il y a? dit une voix derrière elle. La jeune fille, regardant autour d'elle, vit une grenouille dans la fontaine, la tête à la surface de l'eau.

- Oh, c'est toi, dit la jeune fille, ma balle est tombée au fond du puits.

- Je peux t'aider, dit la grenouille, je peux récupérer ta balle. Que m'offriras-tu en échange?

- Tout ce que tu voudras, répondit la princesse. 
- J'aimerais manger dans ton assiette et boire dans ta timbale, continua-t-elle. J'aimerais dormir sur ton lit. Si je récupère ta balle, me promets-tu tout cela, Princesse?

- Oh oui, répondit la princesse, je te le promets." Mais à part elle, elle pensa: "Idiote de grenouille, je ne pourrais jamais vivre avec elle."

Quand la grenouille entendit la promesse, elle descendit dans le puits. Elle fut bientôt remontée à la surface tenant la balle dans sa bouche. Elle la déposa aux pieds de la princesse.

A la vue de la balle la princesse retrouva le sourire. Elle la ramassa et partit en courant.]

Avec des étudiants avancés, l'usage des techniques de conscientisation de type Focus-onForm (réflexion sur les formes et le sens en association) appliquée à des corpus électroniques pourrait pallier le manque d'immersion en milieu anglophone. Le corpus électronique permet d'analyser une multiplicité de données quantifiables grâce à des outils de fouille et d'interroger la fréquence et l'actualité d'expressions lues ou entendues comme je l'ai montré dans l'application au corpus DIDEROT-LONGDALE. On peut utiliser d'autres corpus conversationnels entre locuteurs natifs et non-natifs comme LINDSEI (Louvain International Database of Spoken English Interlanguage, Gilquin, De Cock, \& Granger, 2010) ou des conversations avec des locuteurs natifs tel que LOCNEC (Louvain Corpus of Native Conversations, De Cock, 2004), travailler sur des extraits conversationnels du Contemporary Corpus of American English (COCA) ou du British National Corpus (BNC), ou du Michigan Corpus of Academic Spoken English (MICASE). Voici un autre exemple de focalisation sur des formes anaphoriques utilisé en cours.

MICASE 2000 English Composition Seminar (recorded June 15) (didactisé)

S3: <LAUGH> oh where di- where did that one guy go? $<$ LAUGH $>$ the really funny one.

S1: what one guy?

S4: yeah he was hilarious. <LAUGH>

S2: oh yeah. <LAUGH>

S3: the one who always said the really random stuff.

S4: he sat like right there.

S2: one that was really like, gung ho

S3: <LAUGH $>$ no

S1: oh Justin (cataphoric and anaphoric referent)

S2: yeah.

S4: was that his name?

S1: yeah um he left.

S1: yeah he's very loud wasn't he?

S4: i think he played like tuba or something something that's loud i think he's, [S5: he played what?] partially deaf or something he plays like tuba or something like that.

S1: oh. i think that hmm didn't he tell us that?

S5: (xx) (that stuff)

S4: yeah he's in the music school. [S1: yeah okay] he's definitely a brass person.

$[\mathrm{S} 3:<\mathrm{RIRE}>$ oh où est où est passé ce mec? $<\mathrm{RIRE}>$ le rigolo

S1: qui ce mec?

S4: oui il était à mourir de rire <RIRE>

S2: ah oui <RIRE>

S3: ce mec qui disait toujours des trucs absurdes

S4: il était assis genre juste là

S2: un qui était vraiment genre gung ho

S3: <RIRE> non

S1: ah Justin (référent cataphorique et anaphorique)

S2: ouais

S4: c'est comme ça qu'il s'appelait? 
S1: ouais heu il est parti

S1: ouais il est bruyant t'as pas trouvé?

S4: je crois qu'il jouait un truc genre du tuba ou quelque chose quelque chose de bruyant je crois il est [S5: il jouait de quoi?] en partie sourd ou un truc dans le genre et il joue genre du tuba ou un truc comme ça

S1: ah je pense que heu il nous a pas dit ça?

S5: (xx) (ce truc)

S4: ouais il est à l'école de musique [S1: ouais d'accord] c'est vraiment un cuivre.

L'intérêt de ce corpus oral authentique est qu'il sensibilise les apprenants avancés à divers modes de coréférence, par exemple, le référent explicite (Justin) de plusieurs éléments anaphoriques (that one guy, the really funny one, what one guy, he, his name, a brass person) n'apparaît qu'en milieu de conversation. De même un élément peut avoir plusieurs référents dans le texte, he avec brass person et funny guy; something renvoie à tuba, mais aussi à partially deaf (or something). Dans les conversations familières, le référent n'est parfois même pas mentionné (the really random stuff) car fait appel à la mémoire épisodique collective du groupe.

Divers travaux récents montrent l'intérêt de logiciels pour la conscientisation des questions de référence sur des corpus authentiques : par exemple, Landragin (2014) sur les anaphores et coréférences et la possibilité d'utiliser le logiciel ANALEC pour mettre en évidence des chaînes de coréférence. La visualisation de ces chaînes s'applique essentiellement à des textes écrits de type littéraire dans les exemples qu'il donne (Les Trois Mousquetaires), mais l'intérêt réside dans l'usage de couleurs appliquées aux parties du discours (possessifs, GN défini, GN démonstratif, pronom personnel anaphorique, déictiques, etc.) ainsi qu'aux liens entre les différents maillons dans le texte. Désoyer, Landragin et al (2015) montrent aussi comment à partir d'un corpus de français oral annoté en référence et en coréférence, ANCOR, (ANaphore et Coréférence dans les Corpus Oraux), on peut appliquer le système de résolution automatique de la coréférence CROC (Coreference Resolution for Oral Corpus) pour dégager des caractéristiques de construction de la référence en français, et plus spécifiquement des phénomènes propres à l'oral. Un outil utile pour les corpus en anglais est CoreNLPsuite (développé par The Stanford NLP Group), utilisable dans sa version de démonstration pour travailler la coréférence avec des apprenants avancés. Un exemple de visualisation d'une chaîne de coréférence à partir de The Frog Prince utilisée en cours est présenté en annexe.

\section{Conclusion}

La focalisation sur des questions linguistiquement complexes, des énoncés porteurs d'ajustement intra-subjectif cognitivement complexes, des faits de langue peu saillants à l'oral comme l'anaphore et l'ellipse, permet d'attirer l'attention des apprenants sur leur spécificité, leur rôle dans la construction du discours interactionnel. A écarter ces phénomènes comme moins essentiels à la communication factuelle, à les réduire à des effets de style, qui ne seraient pas indispensables à la compréhension globale d'un message, le risque est grand d'un appauvrissement de la langue conversationnelle authentique en usage pour l'accomplissement de tâches complexes qui nécessitent la mise en oeuvre de plusieurs activités langagières. C'est parce qu'elle n'est " ni routinière, ni automatisée " (CECRL: 19) que la communication orale en langue étrangère requiert de recourir à des stratégies de conscientisation adéquates en milieu 
institutionnel. Quand la transférabilité positive de la langue maternelle à la langue étrangère est faible, une approche contrastive de conscientisation comme celle qui fut développée dans l'approche communicative et cognitive des années 1990 est efficace. Avec l'essor des corpus électroniques, le recours à des pratiques de réflexion sur des corpus authentiques (data-driven awareness) telles que celles proposées en fin d'article contribue à renforcer l'appropriation de formes peu ciblées dans les pratiques pédagogiques traditionnelles. En complément de l'exposition systématique à une langue naturelle et de pratiques de communication orale avec des natifs ou entre nonnatifs, ces stratégies participent d'une triangulation méthodologique actionnelle qui mêle tâches plus ciblées sur l'acquisition linguistique et tâches complexes mobilisant des compétences diverses, langagières et autres.

\section{Annexe}

\section{Chaîne de coréférence pour the Frog Prince : Ipstandford.edu8080/nlp/process}

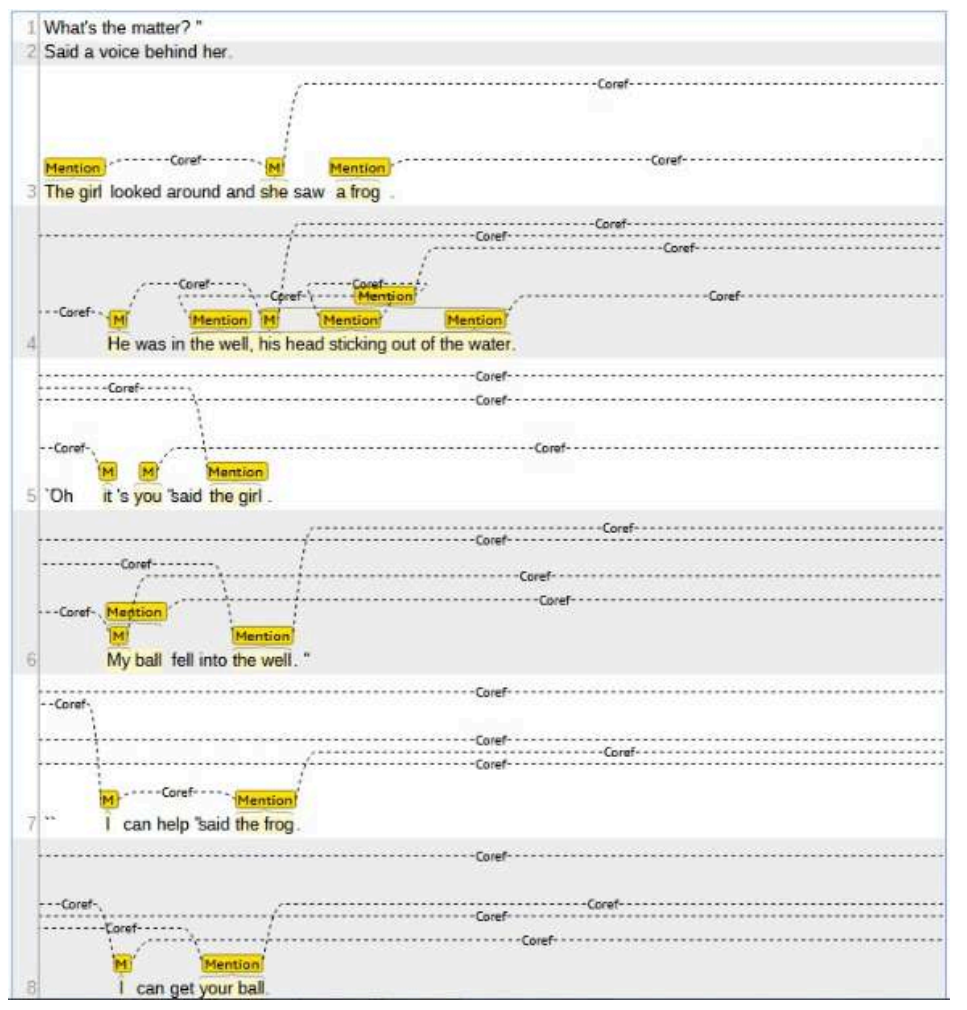

BIBLIOGRAPHIE

BAILLY, D., 1999, Les Mots de la didactique des langues: le cas de l'anglais, lexique, Ophrys, ParisGap. 
BITCHENER, J., 2004, “The Relationship between the Negotiation of Meaning and Language Learning: A Longitudinal Study", Language Awareness, Vol. 13, 2, p. 81-95.

BULTÉ, B., \& HOUSEN, A., 2012, "Defining and Operalising L2 Complexity” in A. Housen, F. Kuiken, \& I. Vedder, Dimensions of L2 Performance and Proficiency, Complexity, Accuracy and Fluency in SLA, chap. 2, , Benjamins, Amsterdam, p. 21-46.

CONSEIL DE L'EUROPE, 2001, Cadre Européen Commun de Référence pour les langues : apprendre, enseigner, évaluer, Didier.

CONSEIL DE L'EUROPE, 2018, Cadre Européen Commun de Référence pour les langues : apprendre, enseigner, évaluer, volume complémentaire avec de nouveaux descripteurs. https://rm.coe.int/ cecr-volume-complementaire-avec-de-nouveaux-descripteurs/16807875d5.

CULIOLI, A., 2002 (2009), Variations sur la linguistique, Entretiens avec Frédéric Fau, préface et notes de Michel Viel, Langres, Klincksieck, Paris.

DE PIETRO, J.-F., MATTHEY, M., et PY, B., 1989, « Acquisition et contrat didactique : les Séquences Potentiellement Acquisitionnelles dans la conversation exolingue ", dans D., Weil et F. Fugier (éds.), Actes du troisième colloque régional de linguistique, Université Louis Pasteur, Strasbourg.

ELLIS, N., 2007, "The Weak Interface, consciousness, and form-focused instruction: mind the doors", in S. Fotos, \& H. Nassaji (eds.) Form-focused Instruction and Teacher Education: Studies in Honour of Rod Ellis, Oxford University Press, Oxford, p. 17-34.

ELLIS, R., 2003, Task-based Language Learning and Teaching, Oxford University Press, Oxford.

ELLIS, R., 2016, “Focus on Form: a Critical Review”, Language Teaching Research, Volume 20, Issue 3, p. 405-428.

DESOYER, A., LANDRAGIN, F. et al, 2015, « Les coréférences à l'oral, une expérience d'apprentissage automatique sur le corpus ANCOR », TAL, vol 55 (2), p. 97-121.

GAONAC'H, D., 1984, « La notion d'interlangue et la psychologie cognitive du langage » dans B. Py (ed.), Acquisition d'une langue étrangère III Encrages, nº spécial, p. 103-109.

GAONAC'H, D. \& LARIGAUDERIE, P., 2000, Mémoire et fonctionnement cognitif, la mémoire de travail, Armand Colin, Paris.

HOUSEN, A. \& SIMOENS, H., 2016, "Introduction: Cognitive perspectives on difficulty and complexity in L2 Acquisition", Studies in Second Language Acquisition, 38 : 63-175.

HUART, R., 2010, Nouvelle Grammaire de l'oral, Ophrys, Paris.

JULIÉ, K. \& PERROT, L., 2008, Enseigner l'anglais, Mise en œuvre du CECRL, Pédagogie actionnelle, Pratiques de classe, Usages des TICE, Hachette, Paris.

LANDRAGIN. F., 2014, Anaphores et coréférences: analyse assistée par ordinateur. Nouvelles perspectives sur l'anaphore. Points de vue linguistique, psycholinguistique et acquisitionnel, Peter Lang, Bern.

LEECH, G., 2000, “Grammars of Spoken English: New outcomes of corpus-oriented research”, Language Learning, 50, 4, p. 675-724.

MCCARTHY, M., 2001, Discourse Analysis for Language Teachers, Cambridge University Press, Cambridge.

MANOÏLOV, P., 2019, « Interaction orale et coopération, un apprentissage interdépendant », Enseigner et apprendre à interagir en langue étrangère : réflexions linguistiques et didactiques, Linx, 79. <https://journals.openedition.org/linx/3784>. 
ORIEZ, S., 2009, Syntaxe de la phrase anglaise, Presses Universitaires de Rennes, Rennes.

PENNEC, B., 2012, « La notion d'ajustement : motivations et application dans le champ des reformulations » dans l'Ajustement dans la TOE d'Antoine Culioli, C. Philippi-Deswelle (ed.), Epilogos, n³, Publication électronique de l'ERIAC, Université de Rouen, p. 181-202.

PENNEC, B., 2017, Réajustement du discours en anglais contemporain, Paris, Ophrys.

SCHMIDT, R., 2001, “Attention” in P. Robinson, (ed.) Cognition and Second Language Instruction, New York, Cambridge University Press, Cambridge, p. 3-32.

SKEHAN, P., 1998, A Cognitive Approach to Language Learning, Oxford University Press, Oxford. WHITE, J., 1998, “Getting the Learner's Attention”, in C. Doughty, \& J. Williams (eds), Focus-on Form in Classroom Second Language Acquisition, Cambridge University Press, Cambridge, p. 85-113.

Manuels de lycée contemporains

New on Target, Belin, 2012

Skylight, Terminale, Hachette, 2012

Freestyle, Terminale, Hachette, 2016

Diversity, Première, Nathan, 2015

Full Swing, Première, Didier, 2016

New Mission, Terminale, Bordas, 2016

New Meeting Point, Première, 2015

Password English, Terminale, Didier, 2012

Corpus électroniques utilisés

DIDEROT-LONGDALE (coord. P. Goutéraux), en cours de publication sur ORTOLANG, <http:// www.clillac-arp.univ-paris-diderot.fr/projets/longdale>

MICASE, English Composition Seminar Transcript ID: SEM300MU100 Academic Division: Publisher: Michigan Corpus of Academic Spoken English, English Language Institute, University of Michigan Recording Date: June 15, 2000.

Autres corpus cités

British National Corpus (BNC)

Contemporary Corpus of American English (COCA)

Louvain International Database of Spoken English Interlanguage, (LINDSEI), 2010, Gilquin, G., De Cock, S., et Granger, S., Presses Universitaires de Louvain-la-Neuve, Belgique.

Louvain Corpus of Native Conversations (LOCNEC), collecté by S. De Cock en 2004, Université de Louvain-la-Neuve, Belgique (non publié).

Logiciels utilisés

The Stanford NLP Group, 2015, CoreNLPsuite, Stanford University, version de démonstration, <http://nlp.stanford.edu:8080/corenlp/>

SCOTT, M., 2016, <Wordsmith7, https://www.lexically.net/wordsmith/> 


\section{RÉSUMÉS}

Cet article traite de la reconnaissance et l'appropriation de structures complexes dans la communication orale par des apprenants d'anglais langue étrangère. Pauvreté lexicale ou nonreconnaissance phonologique sont souvent évoquées comme les obstacles premiers à la compétence de réception orale des non-natifs. L'hypothèse explorée est que certains usages syntaxiques et grammaticaux de l'anglais oral sont à l'origine de hiatus communicationnels entre natifs et non-natifs et limitent la compétence de réception des apprenants d'anglais langue étrangère. L'étude a porté sur des phénomènes d'intercompréhension, ajustement et réajustement discursif observés dans le corpus DIDEROT-LONGDALE, un corpus longitudinal de conversations entre natifs et étudiants de licence de l'université Paris-Diderot (Université de Paris). Les difficultés de compréhension manifestées par les étudiants n'étaient pas seulement dues à la méconnaissance d'éléments lexicaux, à des difficultés de perception orale ou au manque d'attention mais résultaient aussi de la non-reconnaissance de formes orales complexes produites par les assistants anglophones: interrogations non-canoniques ou peu fréquentes, anaphores et formes elliptiques, ou énoncés à propositions multiples. Après l'analyse de ces dysfonctionnements discursifs, l'article discute les stratégies d'inter-ajustement utilisées par les interlocuteurs pour renouer le fil de la conversation et s'interroge sur leur efficacité à long terme pour la consolidation de la compétence linguistique. L'analyse de huit manuels de lycée récents suggère que les apprenants du secondaire ne sont guère exposés aux formes conversationnelles syntaxiques complexes ou peu saillantes. Des stratégies de conscientisation sont ensuite proposées dans une dernière partie avec des techniques de centration sur la Forme à partir de corpus oraux: balayage de corpus conversationnels par des outils de concordance pour l'extraction d'interrogatives, identification de chaines de coréférence (Stanford CORE NLP Suite) ou un ciblage qualitatif d'anaphores.

This article deals with the identification and internalization of complex structures in oral communication by students of English as a foreign language. Limited lexical skills or phonological misperception are often considered as major obstacles to the non-native competence in oral reception. This article hypothesizes that the lack of mastery of some typical grammatical and syntactic structures in use in conversational English disrupt the flow of communication between natives and non-natives and limit the oral competence of foreign language learners. The study explored inter-comprehension, adjustment and re-adjustment phenomena in the DIDEROT-LONGDALE longitudinal corpus of conversations between natives and Bachelor's students from Paris-Diderot University (now University of Paris). The initial hypothesis was that the reception difficulties displayed by the students were not only caused by lexical ignorance, oral misperception or lack of sustained attention. They also resulted from the subjects' failure to grasp complex oral forms produced by the native assistants: non-canonical or rare interrogative structures, anaphoric forms and ellipses as well as long utterances with multiple clauses. After analyzing examples of interactional dysfunction, the article discusses the inter-adjustment strategies used by the interlocutors to resume the conversation. It also questions their long-term efficiency for scaffolding linguistic competence. The analysis of eight recent textbooks used in secondary schools shows that students are seldom explicitly exposed to complex or non-salient conversational features. Consciousness-raising strategies are then suggested in the last part: Focus-on-Form techniques such as the scanning of electronic conversational corpora by concordancing tools to extract interrogatives, the identification of coreference chains (Stanford CORE NLP Suite) or the qualitative targeting of anaphoric elements. 
INDEX

Mots-clés : analyse discursive - anglais langue étrangère (ALE) - corpus - complexité syntaxique - didactique des langues - focalisation sur la forme - interaction orale.

Keywords : corpus - discourse analysis - didactics - English as a Foreign Language (EFL) - form focused instruction - spoken interaction - syntactic complexity.

\section{AUTEUR}

PASCALE GOUTÉRAUX

EA CLILLAC-ARP 3967 Université de Paris 